Neurosurg Focus 17 (6):E3, 2004

\title{
Nonspecific pyogenic spondylodiscitis: clinical manifestations, surgical treatment, and outcome in 24 patients
}

\author{
Sascha Mann, M.D., Michael Schütze, M.D., Steffen Sola, M.D., \\ AND JüRgEN PIEK, M.D. \\ Department of Neurosurgery, University of Rostock, Germany
}

\begin{abstract}
Object. Pyogenic vertebral osteomyelitis is of special interest to neurosurgeons because it often results in acute neurological deterioration and requires a combination of adequate surgical and conservative treatment. The aim of the current study was to evaluate the strategy of a primary surgical approach to this disease.

Methods. A group of 24 patients with the clinical and radiological signs of acute pyogenic spondylodiscitis was prospectively followed from 1998 to 2004 . Of these, 20 had underlying diseases such as diabetes mellitus, chronic alcoholism, and liver cirrhosis. The main causative organism was Staphylococcus aureus. Most infections were localized in the thoracic or lumbar spine (10 cases each); 15 infections were associated with epidural abscesses. Because of a delay in diagnosis, 13 patients presented with neurological deficits on admission.

Patients with a complete or rapidly progressing neurological deficit underwent immediate surgery. In patients with minor or no deficits or in a stable neurological condition, surgery was delayed for 3 to 5 days. This group was treated with immobilization and intravenous antibiotic drugs before surgery. Surgical procedures included ventral, dorsal, and combined approaches in one- or two-stage operations. Antibiotic treatment included the use of broad-spectrum antibiotic drugs delivered intravenously for at least 10 days, followed by orally administered antibiotics for 3 months.

Twenty patients were independent on follow-up review, 15 with no or minor handicaps. Severe septicemia and multiorgan failure developed in two patients, and these two died of their disease. Major complications were mainly due to long-term antibiotic therapy.

Conclusions. Surgical treatment is the modality of choice in patients with acute spinal osteomyelitis. It is especially indicated in patients with progressive or severe neurological deficits and spinal deformity. In experienced hands, surgery is safe and offers the advantages of spinal cord decompression, immediate mobilization, and correction of spinal deformity. The decision whether an anterior or posterior approach should be used must be made on an individual basis.
\end{abstract}

\section{KEY WORDS - spondylodiscitis - spinal abscess • spinal infection • surgical treatment • antibiotic medication - paraplegia}

Nonspecific spontaneous spinal epidural abscesses and spondylodiscitis are rare clinical conditions. ${ }^{19}$ Their incidence, however, appears to be on the rise, which may be attributed to various factors such as the increase in the elderly population, intravenous drug abuse, and the use of epidural catheters for pain therapy. Other important risk factors are diabetes mellitus and impaired immunocompetence (chemotherapy, human immunodeficiency virus infections, or chronic alcoholism)., ${ }^{2,19}$ Because acute pyogenic vertebral osteomyelitis often results in acute neurological deterioration and requires a combination of adequate surgical and conservative treatment, it is of special importance to neurosurgeons.

\footnotetext{
Abbreviations used in this paper: $\mathrm{CRP}=\mathrm{C}$-reactive protein; $\mathrm{CT}=$ computerized tomography; ESR = erythrocyte sedimentation rate; $\mathrm{MR}=$ magnetic resonance; $\mathrm{VB}=$ vertebral body.
}

In the past, antibiotic drugs were the standard (and only) method of treatment for spinal infections. With the advances in modern neurosurgical techniques for spinal instrumentation and fusion, use of these techniques should be reevaluated. Therefore, this study was designed to highlight the effect and the clinical results of primary surgical treatment in cases of acute pyogenic spondylodiscitis.

\section{CLINICAL MATERIAL AND METHODS}

During a 6-year period we prospectively studied 24 patients (14 men and 10 women ranging in age from 37 to 76 years, median age 63 years) who were admitted to our department with clinical and radiological signs of acute pyogenic spondylodiscitis. Pre- and postoperative clinical data, medical records, a full neuroimaging workup (MR images, CT scans, conventional spine x-ray films), and 
laboratory data were analyzed for all patients. Neurological and neuroimaging outcome was assessed at routine intervals, with follow-up visits at 3, 6, 12, and 24 months. Thus, individual follow-up duration ranged from 6 to 24 months. Additionally, all patients were required to answer a structured medical questionnaire (intensity of pain, social and economic situation, activities of daily living).

Patients with a complete or rapidly progressing neurological deficit underwent immediate surgery. In patients with minor or no deficits or a stable neurological condition, surgery was delayed. These patients were treated with immobilization and intravenous antibiotic drugs for up to 3 days before surgery. Surgical procedures included ventral, dorsal, and combined approaches in one- or twostage operations. Antibiotic treatment included the use of intravenous broad-spectrum drugs for at least 10 days, followed by oral antibiotics for 3 months (therapy was eventually continued if inflammation-specific laboratory values [CRP, serum leukocytes, ESR] were still not within the normal range). After hospital discharge, all patients were sent to a specialized rehabilitation facility.

\section{RESULTS}

\section{Clinical Presentation}

Clinical data are summarized in Table 1. None of the 24 patients was free of abnormalities on neurological/laboratory tests. On admission to our department, five patients presented with a complete transversal syndrome (in four of them it had developed within 12 hours before admission; in one patient the deficit had been complete for several days). Another five patients presented with incomplete and progressing para- or tetraparesis (they were unable to walk); three had only minor deficits (radicular); and 11 were neurologically intact.

At least one episode of neck or back pain before admission was reported by all patients, and radicular pain was noted by nine of them. A history of increased temperature $\left(>37^{\circ} \mathrm{C}\right)$ was observed in only nine patients (six of whom had septicemia). Laboratory signs included elevation of CRP, white blood cell count, and ESR in all cases. In most patients laboratory changes were marked by CRP values greater than $40 \mathrm{mg} / \mathrm{L}$ as well as an ESR greater than $60 \mathrm{~mm} /$ hour in 20 patients.

\section{Location of Infection}

Although the majority of patients (21 cases) had pyo-

TABLE 1

Symptoms and clinical data on admission in 24 patients with pyogenic spondylodiscitis

\begin{tabular}{lc}
\hline \hline \multicolumn{1}{c}{ Symptom } & No. of Patients $(\%)$ \\
\hline back/neck pain & $24(100)$ \\
radicular pain & $9(38)$ \\
fever $>38^{\circ} \mathrm{C}$ & $9(38)$ \\
motor deficits & $13(54)$ \\
limb weakness & 3 \\
para- or tetraplegia & 5 \\
para- or tetraparesis & 5 \\
elevated leukocytes \& ESR & $24(100)$ \\
elevated CRP & $24(100)$ \\
CRP $>40 \mathrm{mg} / \mathrm{L}$ & $20(83)$ \\
\hline
\end{tabular}

genic spondylitis at only one VB, there were two patients with bisegmental and one with multisegmental infections. Infections were equally distributed at the thoracic and lumbar spine (10 cases each), and cervical infections were rare (four cases). In 15 cases spondylitis was combined with epidural abscesses. In 12 patients these abscesses were circumscribed at the level of the VB involved, whereas in three patients large epidural abscesses (in up to eight segments) required surgical treatment. All abscesses were located ventrally to the spinal cord.

\section{Predisposing Factors}

The main predisposing factor was diabetes (14 cases); in four patients this was combined with chronic renal failure. Other risk factors included chronic alcoholism (nine cases, four with additional liver cirrhosis), cancer (four), rheumatoid arthritis (one), and others. Nine patients were heavy smokers and another seven had a body mass index greater than $30 \mathrm{~kg} / \mathrm{m}^{2}$. Predisposing factors were lacking in only four patients.

\section{Presumed Source of Infection}

In spite of a careful diagnostic workup (ear, nose, and throat examination including $\mathrm{x}$-ray films of paranasal sinus, echocardiography, and dental examination in four cases) the source of the spinal infection could be presumed in only nine patients (dental origin in four cases, pneumonia in three, septicemia in two). Before or after the spinal procedure, additional operations (for example, tooth extraction) were also performed in these cases.

\section{Causative Organisms}

As shown in Table 2, cultures from intraoperative specimens (bone, disc, and abscess material) were obtained in all cases. In 10 patients bacterial cultures were negative, in another 10 only one causative organism could be identified, and four patients had mixed infections with as many as three different bacteria. The causative organism in one third of our patients was Staphylococcus aureus, and three patients presented with anaerobic infections.

\section{Surgical Treatment}

All patients were treated surgically. The only indication for immediate surgery (that is, directly after establishing the diagnosis of a spinal infection) was a complete or

TABLE 2

Results of intraoperative smears obtained in 24 patients*

\begin{tabular}{lcc}
\hline \multirow{2}{*}{ Bacteria } & \multicolumn{2}{c}{ Infection } \\
\cline { 2 - 3 } S. aureus & Single & Mixed \\
S. epidermidis & 6 & 2 \\
Streptococcus sp. & 0 & 2 \\
Bacteroides fragilis & 2 & 0 \\
Brevibacterium sp. & 1 & 1 \\
Enterococcus sp. & 0 & 1 \\
E. coli & 0 & 1 \\
Lactobacillus & 0 & 1 \\
other & 0 & 1 \\
\hline
\end{tabular}

* Cultures were negative in 10 patients. 
rapidly progressing neurological deficit, which we found in seven patients. In the remaining 17 cases we tried to obtain at least minimal control of the infection by treating it with antibiotic medications before the planned operation, which was scheduled 3 to 5 days after the patient's admission to our department. Unfortunately, two of these 17 patients suffered neurological deterioration during the clinical course and subsequently underwent operation earlier than planned. All patients were initially treated with intravenous antibiotic drugs, which included a combination of dicloxacillin, clindamycin, and gentamicin. The antibiotic therapy was adjusted according to the results of microbiological cultures of blood and intraoperative smears. Intravenous antibiotic medications were continued for at least 10 days or until clinical improvement was noted. After clinical improvement was achieved, all patients received oral antibiotic drugs (clindamycin alone or combined with others) for at least 3 months postoperatively. Long-term antibiotic therapy was discontinued after complete normalization of laboratory parameters.

The aims of our surgical treatment were as follows: decompression of the spinal cord; debridement of the affected VB with removal of all necrotic bone tissue; evacuation of abscess formation if present; and realignment of the deformed spinal column by transpedicular/ intracorporal spondylodesis. Because of the different clinical and radiological presentations of the patients, surgery was adjusted according to the individual case. We performed four ventral procedures in the cervical spine. Procedures in the thoracic and lumbar spine included two ventral, four dorsal, and 14 combined approaches (Table 3 ). If possible, all patients were immediately mobilized after definite stabilization of the spine had been obtained.

\section{Postoperative Outcome}

As shown in Table 4, the hospital stay ranged from 12 to 72 days (mean 33 days). Four patients presenting with initial para- or tetraplegia either died as a result of their infection (two patients) or their condition remained unchanged (two patients). Of the remaining 20 patients, five were mostly dependent, but were able to walk with the help of a crutch. The other 15 patients had only minor or no handicaps and were independent. Only one patient, in whom cervical abscess formation was found and who presented with complete tetraplegia on admission, was able to return to work after treatment for the disease. Transient neurological deterioration was observed in three patients during their hospital stay (one postoperatively, two during the initial conservative treatment). Permanent disability due to neurological worsening was observed in one patient during the initial antibiotic therapy.

TABLE 3

Summary of surgical procedures in 24 patients treated for spinal infection

\begin{tabular}{lccc}
\hline \hline $\begin{array}{l}\text { Treatment } \\
\text { Location }\end{array}$ & Ventral & Dorsal & Combined \\
\hline cervical & 3 & 0 & 1 \\
thoracic & 3 & 3 & 4 \\
lumbar & 0 & 1 & 9 \\
total & 6 & 4 & 14 \\
\hline
\end{tabular}

TABLE 4

Clinical results at last follow-up visit in patients with spinal infection*

\begin{tabular}{ccc}
\hline \hline Outcome Score & Preop & Last FU (min 6 mos) \\
\hline $0 / 5$ & 5 & 2 \\
$1 / 5$ & 2 & 1 \\
$2 / 5$ & 2 & 2 \\
$3 / 5$ & 0 & 1 \\
$4 / 5$ & 1 & 2 \\
$5 / 5$ & 11 & 16 \\
\hline
\end{tabular}

* According to the Medical Research Council scale. Abbreviation: FU = follow up.

\section{Complications of Therapy}

Surprisingly, there were only minor complications that could be attributed to surgical therapy (Table 5). Major complications were only observed as side effects of longterm antibiotic therapy (for example, colitis, renal failure, allergic reactions). Four patients had deep wound infections at the surgical site (two of them had to undergo repeated operation for wound debridement) but they did not require removal of the implanted material for spondylodesis. Further complications of the underlying disease included decubitus ulcers, fungal infections, and pulmonary and urinary tract infections; these were only transient. Despite their severe clinical condition on admission and numerous underlying risk factors, no patient died as a result of the procedure. Nevertheless, two patients died of their underlying disease (that is, severe septicemia developed in one and multiorgan failure in the other).

\section{ILLUSTRATIVE CASES}

\section{Case 1}

This 51-year-old woman presented with a 7-day history of neck and radicular pain. Two days before admission she noted difficulty walking and weakness of the legs. On the day of admission, progressive weakness of the legs developed within hours. On admission to the Department of Neurology she presented with a paraplegia $(0 / 5)$ of the legs, and a paraparesis (4/5) of both arms..$^{29}$ Her upper sensory level was T-9. Her body temperature was $38^{\circ} \mathrm{C}$, leukocyte count was 21,600 cells/ $/ \mathrm{mm}^{3}$, and CRP $159 \mathrm{mg} / \mathrm{L}$. Urgent MR imaging studies and CT scans were obtained, which revealed a ventrally located fluid collection extend-

TABLE 5

Complications during treatment for spinal infection in 24 patients

\begin{tabular}{lc}
\hline \hline \multicolumn{1}{c}{ Complication } & No. of Patients \\
\hline wound infection & 4 \\
allergic reaction & 1 \\
renal failure & 1 \\
colitis & 5 \\
fungal infection & 5 \\
urinary tract infection & 5 \\
pneumonia & 2 \\
decubitus ulcers & 4 \\
death & 2 \\
\hline
\end{tabular}


ing from approximately $\mathrm{C}-2$ to $\mathrm{T}-2$ and a spondylodiscitis at the $\mathrm{C} 3 / 4$ level (Fig. 1A).

Emergency surgery was performed as a ventral discectomy at $\mathrm{C} 3 / 4$ and $\mathrm{C} 6 / 7$, and we found epidural liquid pus at both levels (Fig. 1B). We performed a ventral single fusion with placement of a carbon cage and a ventral plate. Intraoperative smears were positive for $S$. aureus. Postoperatively, the patient underwent a 10-day regimen of intravenous antibiotic drugs (clindamycin, ceftriaxone, gentamicin) and 12 weeks of oral antibiotic medications (clindamycin). At 1 year postsurgery she was neurologically intact and had worked since 8 months after surgery in her former job as a secretary. Follow-up MR images and plain x-ray films (Fig. 1C and D) demonstrated good results.

\section{Case 2}

This 51-year-old man was admitted to the hospital with an 8-week history of persistent fever and back pain. His workup revealed a history of chronic alcoholism and liver cirrhosis as a risk factor. His body temperature at admission was $38.7^{\circ} \mathrm{C}$. His neurological examination showed a complete paraplegia (which had developed after a lumbar puncture at another hospital) with the upper level at L-2. Laboratory results revealed an ESR of $10^{9} \mathrm{~mm} /$ hour, a white blood cell count of 19,700 cells $/ \mathrm{mm}^{3}$, and a CRP value of $215 \mathrm{mg} / \mathrm{L}$. Admission X-ray films, MR images, and CT scans were obtained (Fig. 2A-C) and revealed findings consistent with spondylodiscitis at L2/3 and epidural scar formation.

Initially the patient received intravenous antibiotic medications for 5 days (ciprofloxacin, dicloxacillin, and clindamycin) and additional steroid drugs. After continuous neurological improvement, he underwent surgery. To correct the severe deformity we performed a one-stage ventrodorsal spondylosyndesis (L2-4). As demonstrated on X-ray films obtained 1 year postoperatively (Fig. 2D and E), this operation yielded good results. Ten days postsurgery the patient was transferred to a rehabilitation unit. At Week 12 he was independent, able to walk short distances without assistance, and had complete bladder control.

\section{DISCUSSION}

Acute pyogenic nonspecific spondylodiscitis is a disease that mainly affects the older population ${ }^{2,18}$ or patients with known risk factors. ${ }^{1-3,5,10,18,19,21,30}$ This was also true for our patients, most of whom presented with at least one risk factor, such as diabetes mellitus (58\%) or chronic alcohol or nicotine abuse (38\%). Additionally, $30 \%$ of our patients were overweight, with a body mass index of more than $30 \mathrm{~kg} / \mathrm{m}^{2}$.

The typical patient with acute spondylodiscitis presents with acute pain at the site of the infection, combined with fever. Low-back pain $(63 \%)$ or sciatica $(37 \%)$ were also common symptoms in our patients and have been reported in the literature. ${ }^{10,19}$ Although a history of recent febrile episodes was present in all patients, only two thirds of them actually had an elevated body temperature $\left(>37^{\circ} \mathrm{C}\right)$ on admission. Laboratory examinations specific for infections showed abnormal values in all of our patients (ESR, CRP, white blood cell count). This was not always the case in earlier studies. ${ }^{3,5}$

According to our study and to most other authors, diagnosis of spondylodiscitis is often delayed. ${ }^{1,11}$ Thus the infection spreads, leading to epidural abscess formation and compromising the spinal cord, with subsequent neurological deterioration. Additional epidural abscesses were present in 15 of our patients, causing neurological deficits in 13 of them, with complete tetra- or paraplegia in five cases. The frequency of neurological deficits on admission varies, ${ }^{1,12,19,21}$ but may be as high as $60 \% .^{26}$

The major diagnostic tool in our study was MR imag-
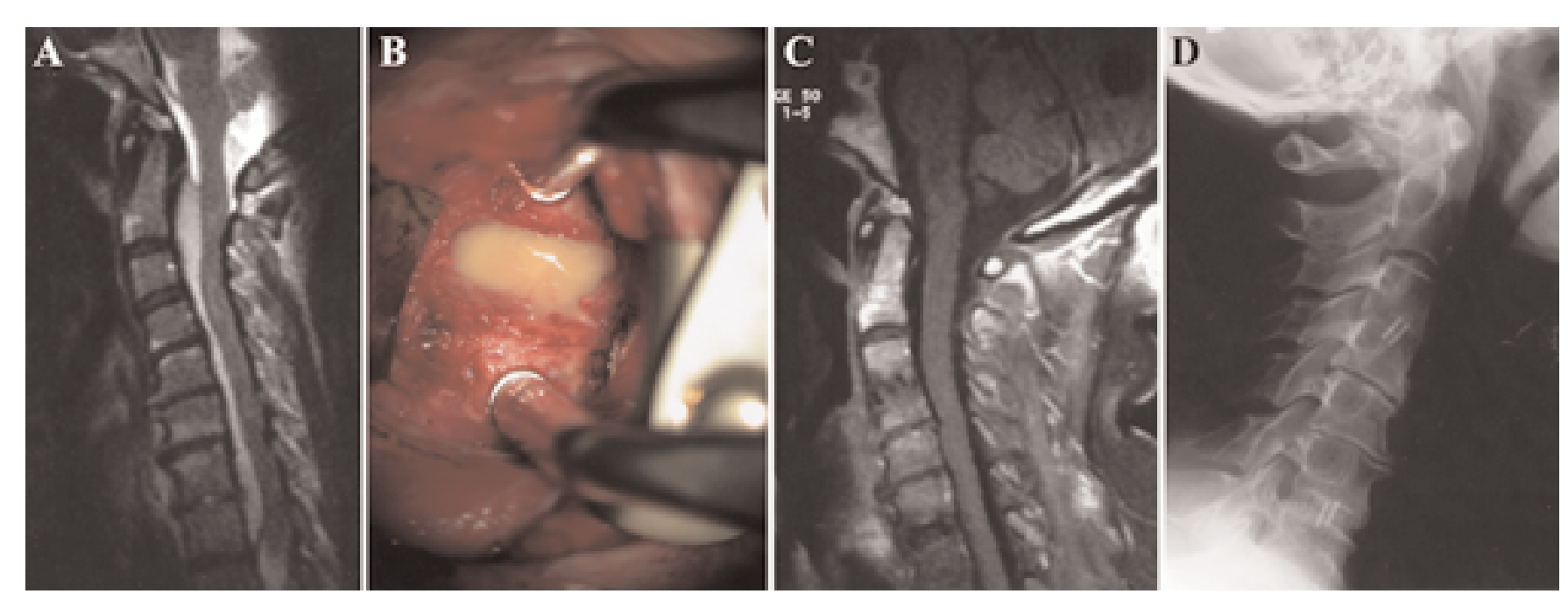

Fig. 1. Case 1. A: Preoperative MR image ( $\mathrm{T}_{1}$-weighted with $\mathrm{Gd}$ contrast) revealing the upper part of an epidural abscess formation extending from C-2 downward due to a spondylodiscitis at C3/4. B: Intraoperative photograph showing epidural pus after removal of the $\mathrm{C} 3 / 4$ disc. C: Postoperative MR image obtained 18 months postsurgery, demonstrating complete removal of the abscess and fusion of the surgically treated segments C3/4 and C6/7. D: Postoperative lateral x-ray films obtained 18 months postsurgery showing resolution of the infection. 

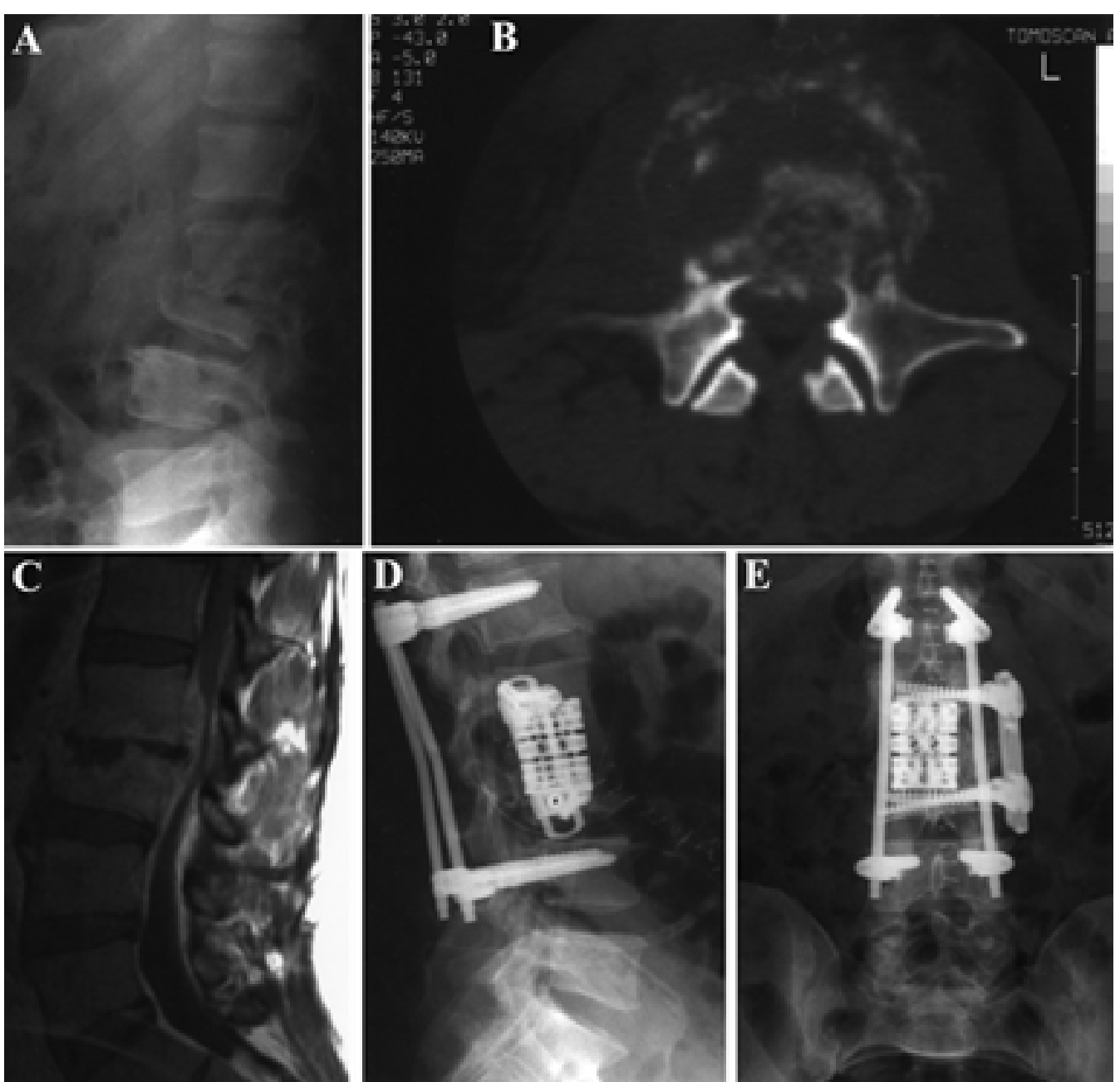

Fig. 2. Case 2. A: Lateral x-ray film of the lumbar spine obtained at admission, showing severe destruction and deformity at the L2-3 level. B: Axial CT scan demonstrating total destruction of the L-3 vertebra due to osteomyelitis. $\mathrm{C}$ : Preoperative MR image ( $\mathrm{T}_{1}$-weighted with $\mathrm{Gd}$ contrast) revealing additional epidural scar formation at the level of infection. D and E: Postoperative X-ray films obtained after spondylodesis at 12-month follow-up review.

ing in all patients, and this modality has proven to be the diagnostic tool of choice. ${ }^{7,10,11,23}$ It has been reported that MR imaging is as sensitive, specific, and accurate as the combination of CT scanning with nuclear medicine studies..$^{30} \mathrm{In}$ acute spondylodiscitis the classic findings on $\mathrm{T}_{1}-$ weighted MR images are low-signal areas of the VB and destruction of the cortical margins of the VB. A high signal in affected areas of the VB and the adjacent discs is typical on $\mathrm{T}_{2}$-weighted MR images. The use of intravenously administered contrast materials is mandatory and pathognomonic in acute inflammatory episodes, and it is of special diagnostic value if additional epidural abscesses are suspected. ${ }^{27}$ Contrast enhancement may persist for several weeks or months.

Spondylodiscitis may be specific or nonspecific. The incidence of specific infections such as tuberculosis is highly dependent, however, on the status of development in the individual country. In our region (northeast Germany) we have only encountered two cases of spinal tuberculosis (which were not included in the present material) during our study.
The least-invasive method available to obtain a bacteriological diagnosis is the blood culture. These, however, are positive in only $35 \%$ of cases. ${ }^{6,13,22}$ Although some authors favor needle biopsy sampling of the affected vertebrae to establish the diagnosis ${ }^{11,24}$ we rarely perform such procedures. In the largest study so far, six needle biopsy procedures led to identification of the causative organism in only $53 \%$ of cases. Needle biopsy sampling followed by conservative treatment may be an option in early cases of spinal infection. ${ }^{19}$ Because most of our patients presented with late infections, neurological compromise, spinal deformity, and epidural abscess formation, direct surgical treatment was the favored approach, with bacterial smears obtained during the procedure.

Tissue cultures were obtained in all patients, and from these cultures bacterial isolates were identified in 14 of 24 patients. This low rate may be the result of preoperative antibiotic therapy or inadequate culture treatment and long transportation time interfering with growth of anaerobic bacteria.

The most commonly isolated organism in our study was 
S. aureus, which was found in $30 \%$ of our patients. This coincides with the current literature, in which $S$. aureus accounts for 42 to $84 \%$ of all spinal infections, ${ }^{12,16,24,26}$ followed by Gram-negative organisms and anaerobic bacteria. ${ }^{19}$

Surgeons are generally reluctant to implant foreign material in infected parts of the body. Thus, there are only limited studies in which instrumentation is used to treat spinal infections. With improved surgical techniques, the use of new broad-spectrum antibiotic medications, and the development of better materials for spondylodesis, numerous authors nowadays favor a primary surgical approach to treat spinal infections. ${ }^{4,8,14,15,17,20,25,28}$ The main advantage of the surgical approach is that it addresses all aspects of the disease: debridement of the infected tissue; identification of the causative organism, enabling specific antibiotic therapy; correction of the eventually deformed spine; and decompression of the spinal cord. Surgical treatment also allows immediate mobilization, thus avoiding the side effects of long-term bed rest, such as deep venous thrombosis.

Whether an anterior or a posterior approach should be used is still a matter of discussion. ${ }^{8,9,12,14}$ It is our belief that general statements on whether either of these approaches is superior cannot be made: surgery for spinal infections has to be performed on an individual basis. In our opinion, the question in such cases is this: which surgical approach is the best for addressing all aspects mentioned earlier (decompression, stabilization, debridement) in any given patient? With the exception of the cervical spine, where a ventral approach usually is sufficient to address the disease, this explains the wide variety of surgical approaches used in our series.

Most patients improved significantly after surgery: besides improvement of their general condition (fever, infection parameters) neurological recovery was observed in most of them. At their last follow-up visit, 15 patients were independent; even one patient who had suffered a complete paraplegia (Case 2) was able to walk and to return to work. Four who were initially para- or tetraplegic did not recover, however. Two of them died of their disease, which underlines the need for earlier diagnosis. This is also in agreement with all of the literature cited in this article.

Side effects were mainly due to long-term antibiotic therapy and were sometimes severe. Therefore, future studies should focus especially on improvement of antibiotic regimens in such cases.

\section{CONCLUSIONS}

Summarizing our favorable results, surgical treatment is the method of choice in patients with acute spinal osteomyelitis. It is especially indicated in patients with progressive or severe neurological deficits and spinal deformity. In cases of epidural abscesses, even patients with a severe neurological deficit can make a full recovery. Surgery is safe in experienced hands and offers the advantages of spinal cord decompression, immediate mobilization, and correction of spinal deformity.

\section{References}

1. Belzunegui J, Del Val N, Intxausti JJ, et al: Vertebral osteomyelitis in northern Spain. Report of 62 cases. Clin Exp Rheumatol 17:447-452, 1999

2. Belzunegui J, Intxausti JJ, De Dios JR, et al: Haematogenous vertebral osteomyelitis in the elderly. Clin Rheumatol 19: 344-347, 2000

3. Calvo JM, Ramos JL, Garcia F, et al: [Pyogenic and non-pyogenic vertebral osteomyelitis: descriptive and comparative study of a series of 40 cases.] Enferm Infecc Microbiol Clin 18:452-456, 2000 (Spn)

4. Castilla JM, Martin V, Rodriguez-Salazar A: [Surgical treatment of patients with spinal infection.] Neurocirugia 13: 101-109, 2002 (Spn)

5. Colmenero JD, Jimenez-Mejias ME, Sanchez-Lora FJ, et al: Pyogenic, tuberculous, and brucellar vertebral osteomyelitis: a descriptive and comparative study of 219 cases. Ann Rheum Dis 56:709-715, 1997

6. Cotty P, Fouquet B, Pleskof L, et al: Vertebral osteomyelitis: value of percutaneous biopsy. 30 cases. J Neuroradiol 15: 13-21, 1988

7. Cusmano F, Calabrese G, Bassi S, et al: [Radiologic diagnosis of spondylodiscitis: role of magnetic resonance.] Radiol Med 100:112-119, 2000 (Ita)

8. Eysel P, Hopf C, Vogel I, et al: Primary stable anterior instrumentation or dorsoventral spondylodesis in spondylodiscitis? Results of a comparative study. Eur Spine J 6:152-157, 1997

9. Faraj AA, Webb JK: Spinal instrumentation for primary pyogenic infection report of 31 patients. Acta Orthop Belg 66: 242-247, 2000

10. Fica A, Bozan F, Aristegui M, et al: [Spondylodiscitis. Analysis of 25 cases.] Rev Med Chil 131:473-482, 2003 (Spn)

11. Flamme $\mathrm{CH}$, Lazoviae D, Gosse F, et al: [MRI in spondylitis and spondylodiscitis.] Orthopade 30:514-518, 2001 (Ger)

12. Hadjipavlou AG, Mader JT, Necessary JT, et al: Hematogenous pyogenic spinal infections and their surgical management. Spine 25:1668-1679, 2000

13. Hitchon PW, Osenbach RK, Yuh WT, et al: Spinal infections. Clin Neurosurg 38:373-387, 1992

14. Hopf C, Meurer A, Eysel P, et al: Operative treatment of spondylodiscitis-what is the most effective approach? Neurosurg Rev 21:217-225, 1998

15. Khan IA, Vaccaro AR, Zlotolow DA: Management of vertebral diskitis and osteomyelitis. Orthopedics 22:758-765, 1999

16. Kilani B, Ammari L, Tiouiri H, et al: [Bacterial spondylodiskitis: pyogenic or tuberculous?] Ann Med Interne 152:236-241, 2001 (Fre)

17. Klockner C, Valencia R: Sagittal alignment after anterior debridement and fusion with or without additional posterior instrumentation in the treatment of pyogenic and tuberculous spondylodiscitis. Spine 28:1036-1042, 2003

18. Krogsgaard MR, Wagn P, Bengtsson J: Epidemiology of acute vertebral osteomyelitis in Denmark: 137 cases in Denmark 1978-1982, compared to cases reported to the National Patient Register 1991-1993. Acta Orthop Scand 69:513-517, 1998

19. Levi ADO, Sonntag VKH: Pyogenic vertebral osteomyelitis, in Osenbach RK, Zeidman SM (eds): Infections in Neurological Surgery: Diagnosis and Management. Philadelphia: Lippincott-Raven, 1999, pp 257-263

20. Linhardt O, Kruger A, Krodel A: [First results of anterior versus posterior instrumentation-fusion in the treatment of spondylodiscitis.] Z Orthop Ihre Grenzgeb 142:73-78, 2004 (Ger)

21. Nolla JM, Ariza J, Gomez-Vaquero C, et al: Spontaneous pyogenic vertebral osteomyelitis in nondrug users. Semin Arthritis Rheum 31:271-278, 2002

22. Osenbach RK, Hitchon PW, Menezes AH: Diagnosis and man- 
agement of pyogenic vertebral osteomyelitis in adults. Surg Neurol 33:266-275, 1990

23. Ozaksoy D, Yucesoy K, Yucesoy M, et al: Brucellar spondylitis: MRI findings. Eur Spine J 10:529-533, 2001

24. Phadke DM, Lucas DR, Madan S: Fine-needle aspiration biopsy of vertebral and intervertebral disc lesions: specimen adequacy, diagnostic utility, and pitfalls. Arch Pathol Lab Med 125:1463-1468, 2001

25. Przybylski GJ, Sharan AD: Single-stage autogenous bone grafting and internal fixation in the surgical management of pyogenic discitis and vertebral osteomyelitis. J Neurosurg Spine 94:1-7, 2001

26. Rath SA, Neff U, Schneider O, et al: Neurosurgical management of thoracic and lumbar vertebral osteomyelitis and discitis in adults: a review of 43 consecutive surgically treated patients. Neurosurgery 38:926-933, 1996
27. Rodiek SO: [Diagnostic methods in spinal infections.] Radiologe 41:976-986, 2001 (Ger)

28. Schinkel C, Gottwald M, Andress HJ: Surgical treatment of spondylodiscitis. Surg Infect 4:387-391, 2003

29. Seddon HJ: Peripheral Nerve Injuries. Medical Research Council Special Report Series 282. London: Her Majesty's Stationary Office, 1954

30. Tali ET: Spinal infections. Eur J Radiol 50:120-133, 2004

Manuscript received October 21, 2004.

Accepted in final form November 4, 2004.

Address reprint requests to: Michael Schütze, M.D., Department of Neurosurgery, University of Rostock, Schillingallee 35, 18057 Rostock, Germany. email: michael.schuetze@med.uni-rostock.de. 